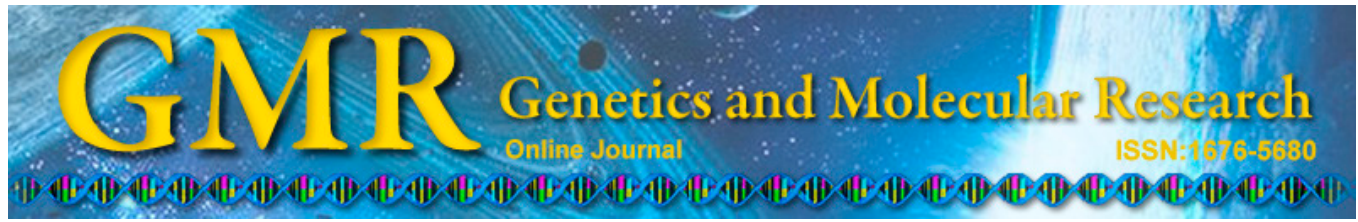

\title{
Differential expression of glypican-3 (GPC3) in lung squamous cell carcinoma and lung adenocarcinoma and its clinical significance
}

\author{
X. Yu, Y. Li, S.W. Chen, Y. Shi and F. Xu \\ Oncology Department, Gongli Hospital of Pudong New Area, \\ Shanghai, China \\ Corresponding author: Y. Li \\ E-mail: chenshi_wen3@163.com \\ Genet. Mol. Res. 14 (3): 10185-10192 (2015) \\ Received January 23, 2015 \\ Accepted June 11, 2015 \\ Published August 28, 2015 \\ DOI http://dx.doi.org/10.4238/2015.August.28.2
}

\begin{abstract}
In this study, we examined the expression of glypican-3 (GPC3) in the 2 most common histological types of lung cancer, squamous cell carcinoma and adenocarcinoma, and explored the relationship between GPC3 expression and the prognosis of these 2 types of lung cancers. Lung cancer tissues and paracancerous tissues were collected from a total of 60 patients with lung squamous cell carcinoma or lung adenocarcinoma. GPC3 gene and protein expression in the tissue samples was examined using fluorescence-based realtime quantitative polymerase chain reaction, immunohistochemistry, and western blot analysis. In addition, the serological levels of GPC3 protein in lung cancer patients were analyzed using enzyme-linked immunosorbent assays. The overall expression of GPC3 protein in lung cancer was 45\% (21/60). No GPC3 expression was detected in paracancerous lung tissues. Positive expression of GPC 3 protein in lung squamous cell carcinoma was significantly higher than that in lung adenocarcinoma (70 vs 20\%, P < 0.001). Among GPC3-positive lung squamous cell carcinoma and lung adenocarcinoma samples, samples collected from patients with lymph node metastasis and patients
\end{abstract}


with poorly differentiated cancer exhibited more pronounced GPC-3 expression. GPC3 protein expression was significantly higher in lung squamous cell carcinoma than in lung adenocarcinoma. GPC3 may be a candidate marker for detecting lung squamous cell carcinoma.

Key words: Glypican-3; Lung adenocarcinoma; Lung squamous cell carcinoma

\section{INTRODUCTION}

Glypican-3 (GPC3) is a membrane-bound proteoglycan that was discovered in patients with Simpson-Golabi-Behmel syndrome in 1996 by Pilia et al. (1996). GPC3 is a $66,000-\mathrm{kDa}$ protein belonging to the heparan sulfate proteoglycan family. This protein is distributed on the cell surface and in the extracellular matrix. Mutation and abnormal expression of GPC3 protein have been associated with malignant tumors (Umezu et al., 2010; Kim and Kang, 2013; Greten et al., 2013; Feng and Ho, 2014). GPC3 is a member of the superfamily of membrane-bound heparan sulfate proteoglycans. GPC3 is a complex glycoconjugate consisting of covalently linked proteins, lipids, and carbohydrates. The carboxyl terminus of GPC 3 is anchored to the cytoplasmic membrane via a glycosyl phosphatidylinositol linkage. Through interactions with the extracellular matrix, cell growth factors, and proteases, GPC3 regulates the proliferation, differentiation, adhesion, and metastasis of the tumor cells. The amino terminus of GPC 3 is also observed in the soluble form of the GPC3 protein, which can be secreted into the peripheral blood by tissues and cells (Aviel-Ronen et al., 2008). Recent studies have confirmed that GPC3 is closely related to the development, progression, and prognosis of malignant tumors, such as hepatocellular carcinoma, and may serve as a valuable tumor marker for the early diagnosis of hepatocellular carcinoma (Magistri et al., 2013). Correlations have been observed between GPC3 and the degree of tumor differentiation, tumor metastasis, and tumor recurrence. In addition, GPC3 expression has been found to be closely related to tumor size (Xu et al., 2013).

Lung cancer is one of the most common malignancies. The prognosis of lung cancer patients is related to the pathological type of the lung cancer. To date, few studies have examined the relationship between GPC3 and lung cancer. Lin et al. (2012) observed that lung squamous cell carcinoma tissues express a significantly higher level of GPC3 protein compared with paracancerous tissues, indicating the potential diagnostic value of GPC3 in lung squamous cell carcinoma. However, GPC 3 expression in other pathological types of lung cancer remains unclear. In this study, we investigated differences in GPC3 expression between lung squamous cell carcinoma and lung adenocarcinoma.

\section{MATERIAL AND METHODS}

\section{Sample source and handling}

Lung cancer tissues and paracancerous tissues (tissues $2 \mathrm{~cm}$ from the tumor margins) were collected from 60 primary lung cancer patients who underwent surgical resection in the Oncology Department of Gongli Hospital of Pudong New Area, Shanghai, between May 2009 and September 2012, including 30 cases of lung squamous cell carcinoma and 30 cases of lung 
adenocarcinoma. Tissue samples were $1 \times 1 \mathrm{~cm}$ in size and were collected immediately after surgery. All tissue samples were stored in liquid nitrogen for future assays. In addition, $5 \mathrm{~mL}$ fasting venous blood was collected from all patients included in the present study to determine serological GPC3 levels.

\section{Experimental approach for determining GPC3 levels}

The serologic concentrations of GPC3 were assessed using an enzyme-linked immunosorbent assay kit purchased from R\&D Systems, Inc. (Minneapolis, MN, USA) according to manufacturer instructions. The absorbance value (A value) in each well at the dual-wavelength of 450/630 nm was measured using the BioRad-500 ELISA reader (Hercules, CA, USA). Using the 4-parameter logistic curve fit generated using the software, a standard curve for GPC3 protein concentration was established. The concentration of GPC3 protein in each serum sample was determined by comparing the A value of the sample to the standard curve.

\section{Immunohistochemistry analysis of GPC3 protein expression}

Anti-GPC3 antibody was purchased from Santa Cruz Biotechnology, Inc. (Santa Cruz, CA, USA). The antibody titer was 1:200. All tissue samples were fixed in 10\% formalin and embedded in paraffin. Next, $4-\mu \mathrm{M}$ serial sections were prepared, placed onto glass microscope slides, and incubated in a $65^{\circ} \mathrm{C}$ oven for $2 \mathrm{~h}$ to melt the paraffin. The tissue sections were then dewaxed, re-hydrated, and subjected to antigen retrieval for $15 \mathrm{~min}$ in sodium citrate buffer. Following antigen retrieval, the tissue sections were treated with hydrogen peroxide for $15 \mathrm{~min}$ to block endogenous peroxidase activity. The sections were incubated with anti-GPC3 antibody overnight at $4^{\circ} \mathrm{C}$, followed by 30 min-incubation at room temperature. The sections were then incubated with a primary antibody enhancer at room temperature for $20 \mathrm{~min}$, rinsed, incubated with horseradish peroxidase-conjugated secondary antibody in a $37^{\circ} \mathrm{C}$ water bath for $30 \mathrm{~min}$, and subjected to 3,3'-diaminobenzidine chromogenic staining and hematoxylin counterstaining.

\section{Evaluation of immunohistochemical staining results}

The results of immunohistochemical staining were evaluated using a double-blind method. GPC3 protein was expressed primarily in the cytoplasm and stained brownish yellow. The background was virtually colorless. GPC 3 immunostaining was scored according to the quantitative scoring system described by Tanaka et al. (2011). Briefly, 5 high-power microscopic fields (400X) were randomly selected in each tissue section, and 200 cancer cells were observed in each high-power field. The total number of cells and number of GPC3-positive cells were counted, and each field was scored based on the percentage of GPC3-positive cells. The scores were as follows: 1 point, $\leq 5 \%$ positive cells; 2 points, $>5-25 \%$ positive cells; 3 points, $>25-50 \%$ positive cells; and 4 points, $>50 \%$ positive cells. In addition, a second score was assigned to each field for staining intensity. The scores were as follows: 0 points, colorless; 1 point, light yellow; 2 points, brownish-yellow; and 3 points, dark brown. The final score for each tissue section was obtained by multiplying the 2 scores of each field and then calculating the average of the 5 selected fields. Final scores were categorized into 4 ranks: -, 0-1 point; +, 2-4 points; ++, 5-8 points; and +++, 9-12 points. - was considered negative; + , weakly positive; ++ , moderately positive; and +++ , strongly positive. 


\section{Reverse transcription polymerase chain reaction (RT-PCR) analysis}

Expression levels of GPC3 mRNA in the tissue samples were analyzed using RT-PCR analysis. The primer sequences were as follows: forward primer, 5'-TGCTCTTACTGCCAG GGACT-3'; reverse primer, 5'-GCTTTCCTGGATTCTTCTGG-3'. PCR amplification conditions were as follows: $25-\mu \mathrm{L}$ PCR mix containing $5 \mu \mathrm{L}$ cDNA; pre-denaturation at $94^{\circ} \mathrm{C}$ for 5 min; 30 cycles of denaturation at $94^{\circ} \mathrm{C}$ for $30 \mathrm{~s}$, annealing at $58^{\circ} \mathrm{C}$ for $1 \mathrm{~min}$, and extension at $72^{\circ} \mathrm{C}$ for $45 \mathrm{~s}$; and final extension at $72^{\circ} \mathrm{C}$ for $10 \mathrm{~min}$. PCR products $(5 \mu \mathrm{L}$ each) were analyzed by electrophoresis on a $1.5 \%$ agarose gel containing $0.5 \mu \mathrm{g} / \mathrm{mL}$ ethidium bromide.

\section{Total protein extraction}

A total of $50 \mathrm{mg}$ frozen tissues were ground and transferred to 1.5-mL Eppendorf tubes. The tissues were mixed with $1 \mathrm{~mL}$ lysis buffer, lysed on ice for $30 \mathrm{~min}$, and sonicated for $30 \mathrm{~s}$. Sonication was performed in an ice bath with a 6-s pause between 6-s sonication bursts. Air bubbles were carefully avoided during the sonication process. Lysates were centrifuged at $15,000 \mathrm{~g}$ and $4^{\circ} \mathrm{C}$ for $1 \mathrm{~h}$. Supernatants were collected, aliquoted $(100 \mu \mathrm{L} / \mathrm{vial})$, and stored at $-80^{\circ} \mathrm{C}$. Protein concentrations were determined using the bicinchoninic acid protein assay kit according to the manufacturer instructions.

\section{Western blot}

Protein preparations corresponding to different histological types of lung cancer tissues were obtained by mixing total proteins exacted from each type of cancer tissue samples (10 $\mu \mathrm{g}$ protein per tissue sample). Similarly, protein preparations corresponding to paracancerous normal tissues were derived from the mixture of total proteins exacted from each paracancerous tissue sample (10 $\mu \mathrm{g}$ protein per tissue sample). These samples were subjected to sodium dodecyl sulfate polyacrylamide gel electrophoresis, with $40 \mu \mathrm{g}$ protein loaded in each lane. After electrophoresis, the proteins were transferred to a polyvinylidene difluoride membrane. The membrane was blocked, probed with primary antibodies at $4^{\circ} \mathrm{C}$ overnight, and incubated with horseradish peroxidase-conjugated secondary antibodies at $37^{\circ} \mathrm{C}$ for $1 \mathrm{~h}$. After washing, target proteins were visualized using enhanced chemiluminescence reagents and autoradiography. The relative content of GPC3 protein was expressed as the gray-scale ratio of GPC3 to glyceraldehyde 3-phosphate dehydrogenase. Each experiment was repeated 3 times.

\section{Statistical analysis}

A database was created using the SPSS 13.0 software (SPSS, Inc., Chicago, IL, USA). Measurement data were analyzed using the $t$ test. Count data were analyzed using the chisquare test. P values less than 0.05 were considered to be statistically significant.

\section{RESULTS}

\section{Comparison of serological GPC3 levels}

As shown in Table 1, serological GPC3 levels were significantly higher in patients 
with lung squamous cell carcinoma than in patients with lung adenocarcinoma. The difference was statistically significant $(\mathrm{P}<0.05)$.

Table 1. Comparison of GPC 3 concentration between lung squamous cell carcinoma and lung adenocarcinoma.

\begin{tabular}{llcc}
\hline Groups & $\mathrm{N}$ & GPC-3 (ng/mL) & P value \\
\hline Lung squamous cell carcinoma & 30 & $889.3 \pm 443.6$ & $<0.0001$ \\
Lung adenocarcinoma & 30 & $232.6 \pm 122.8$ & \\
\hline
\end{tabular}

\section{Expression of GPC3 mRNA in lung cancer tissues}

GPC3 mRNA was expressed at significantly higher levels in lung cancer tissues than in paracancerous lung tissues. Among lung cancer tissues, lung squamous cell carcinoma tissues showed significantly higher levels of GPC3 mRNA expression compared with lung adenocarcinoma tissues $(\mathrm{P}<0.05)$ (Figure 1).

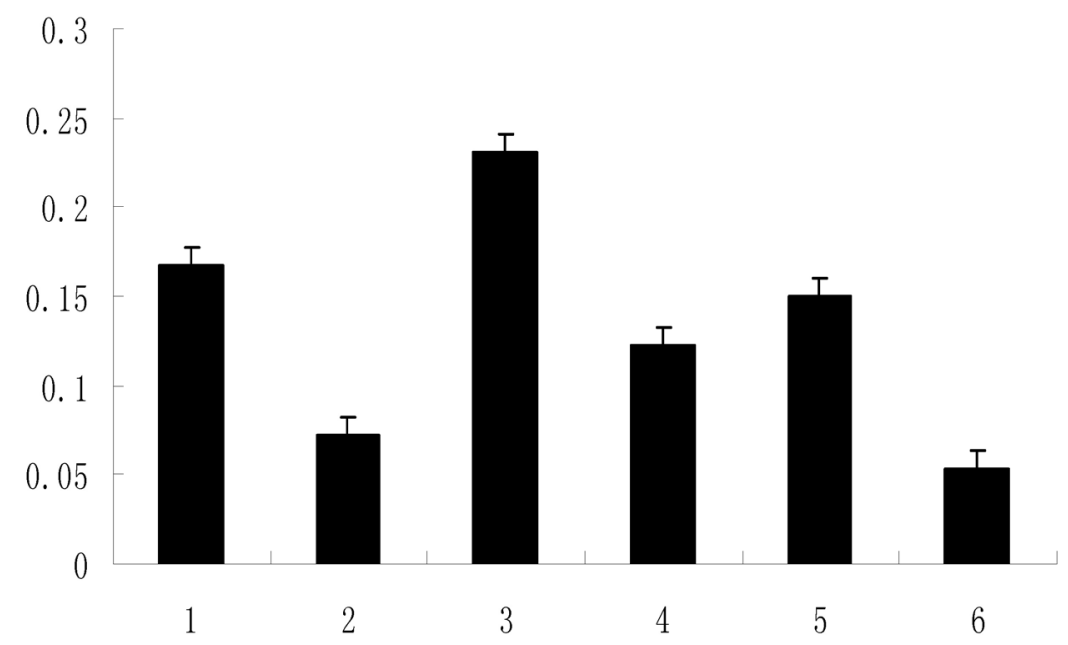

Figure 1. Expression of GPC3 protein in lung cancer and in the adjacent tissues (RT-PCR). Column 1: Lowdifferentiated adenocarcinoma; Column 2: high-differentiated adenocarcinoma; Column 3: low-differentiated squamous cell carcinoma; Column 4: high-differentiated squamous cell carcinoma; Column 5: lymph nodes; Column 6: adjacent tissues.

\section{Immunohistochemistry}

The overall expression rate of GPC3 protein in lung cancer was $45 \%(27 / 60)$. The relationship between the GPC3 expression rate and clinical features such as smoking status, histological types of lung cancer, and clinical stage of the cancer is summarized in Table 2. GPC3 expression was undetectable in paracancerous lung tissues. The positive expression rate of GPC3 protein was significantly higher in lung squamous cell carcinoma tissue than in lung adenocarcinoma tissue (70 vs 20\%, $\mathrm{P}<0.010)$. Among GPC3-positive lung squamous cell carcinoma and lung adenocarcinoma samples, the samples collected from patients with lymph node metastasis and patients with poorly differentiated cancer exhibited more significant GPC3 expression (Figure 2). 
Table 2. Clinical data of patients with different GPC3 expression profiles.

\begin{tabular}{lccc}
\hline & GPC3 positive cases & GPC3 negative cases & Total cases \\
\hline Smoking & & & 35 \\
Yes & 14 & 21 & 25 \\
No & 13 & 12 & 18 \\
Staging & 5 & 12 & 15 \\
I & 6 & 10 & 15 \\
II & 11 & 4 & 12 \\
III & 5 & 7 & 30 \\
IV & & 24 & 30 \\
Pathology & 6 & 9 & \\
Adenocarcinoma & 21 & & \\
Squamous cell carcinoma & & & \\
\hline
\end{tabular}

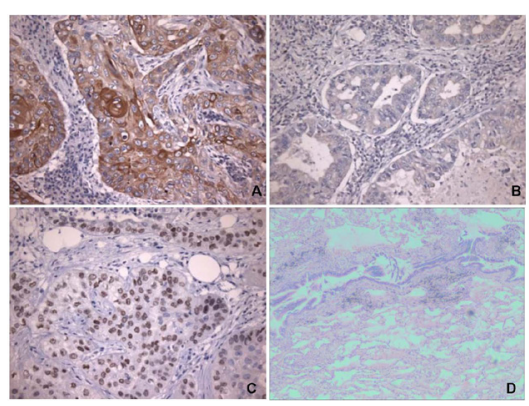

Figure 2. GPC3 expression in lung squamous cell carcinoma (A), lung adenocarcinoma (B), lung adenocarcinoma with lymph node metastasis (C), and adjacent tissues (D) (Immunohistochemistry, original magnification: 200X).

\section{Examination of GPC3 protein expression by western blot analysis}

Consistent with the results of western blot analysis, immunohistochemical analysis revealed that expression levels of GPC3 were significantly higher in lung squamous cell carcinoma tissues than in lung adenocarcinoma tissues. The lowest GPC 3 expression was observed in paracancerous lung tissues (Figure 3).
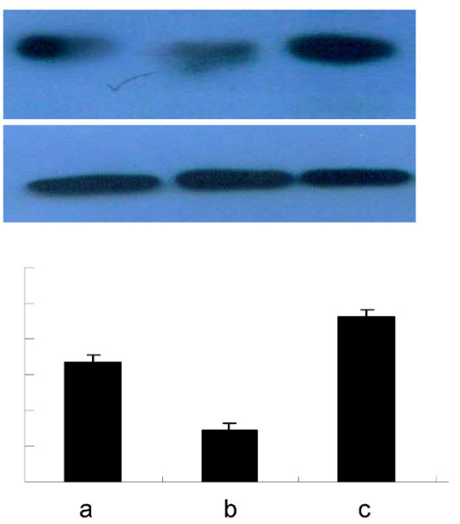

Figure 3. Expression of GPC3 protein in lung cancer and in the adjacent tissues (western blot). a = lung adenocarcinoma, $\mathrm{b}=$ adjacent tissues, and $\mathrm{c}=$ lung squamous cell carcinoma. 
GPC3 and lung cancer

\section{DISCUSSION}

In this study, we demonstrated that the expression of the GPC3 gene and protein was increased considerably in lung cancer tissues. Compared with lung adenocarcinoma tissues, lung squamous cell carcinoma tissues expressed significantly higher levels of GPC3.

The expression of GPC3 differs in various cancer tissues. Previous studies have demonstrated that GPC3 expression is downregulated in gastric (Ushiku et al., 2009), breast (Xiang et al., 2001), and lung cancers (Kim et al., 2003), but is upregulated in liver cancer and melanoma (Komori et al., 2010). The present study demonstrated that the overall expression rate of GPC 3 protein in lung cancer was $45 \%$. In addition, a significant difference in the GPC3 expression rate was detected between the 2 most common pathological types of lung cancer. The GPC 3 protein expression rate was $70 \%$ in lung squamous cell carcinoma, whereas the corresponding rate was only $20 \%$ in lung adenocarcinoma. These results indicate that GPC3 protein can serve as a marker for the early detection of lung squamous cell carcinoma.

In lung adenocarcinoma, the expression rate of GPC3 protein was very low. The difference in GPC3 expression between lung squamous cell carcinoma and lung adenocarcinoma suggests that GPC3 plays distinct or even completely opposite roles in different histological types of lung cancer. The differential expression of GPC3 can be used to differentiate and diagnose lung squamous cell carcinoma and lung adenocarcinoma. Basic research has indicated that GPC3 regulates the proliferation, differentiation, adhesion, and migration of tumor cells by modulating the insulin-like growth factor and wingless/integrated signaling pathways (Avanesov et al., 2012; Sakane et al., 2012; Pez et al., 2013; Dwivedi et al., 2013; Avanesov and Blair, 2013), thus playing important roles in tumor growth and metastasis. Stronger GPC3 expression has been correlated with increased growth and metastatic potential (Tanaka et al., 2011; Gao and Ho, 2011). We observed that among GPC3-positive lung squamous cell carcinoma and lung adenocarcinoma samples, the samples collected from patients with lymph node metastasis or poorly differentiated cancer exhibited more pronounced GPC-3 expression. Our results indicated that GPC3 functions as an important molecular marker in the metastasis of lung cancer to lymph nodes.

In summary, GPC3 protein expression is closely related with the development and progression of lung cancer. GPC3 is differentially expressed between the 2 most common pathological types of lung cancer, squamous cell carcinoma and adenocarcinoma. GPC3 expression was significantly higher in lung squamous cell carcinoma, suggesting that GPC3 can serve as a marker for detecting lung squamous cell carcinoma. GPC3 protein expression varies among different histological types of lung cancer. The relationship between GPC3 and lung cancers is complicated. GPC3 may play distinct roles in lung squamous cell carcinoma and lung adenocarcinoma because of the presence of different signaling pathways or growth factors in the 2 types of lung cancer.

\section{ACKNOWLEDGMENTS}

Research supported by the Municipal Commission of Health and Family Planning on Pudong New Area (\#PW2011A-51) and the Incidental Subject of Pudong New Area's Key Discipline (\#PWZx2014-08). 


\section{REFERENCES}

Avanesov A and Blair SS (2013). The Drosophila WIF1 homolog Shifted maintains glypican-independent Hedgehog signaling and interacts with the Hedgehog co-receptors Ihog and Boi. Development 140: 107-116.

Avanesov A, Honeyager SM, Malicki J and Blair SS (2012). The role of glypicans in Wnt inhibitory factor-1 activity and the structural basis of Wifl's effects on Wnt and Hedgehog signaling. PLoS Genet. 8: e1002503.

Aviel-Ronen S, Lau SK, Pintilie M, Lau D, et al. (2008). Glypican-3 is overexpressed in lung squamous cell carcinoma, but not in adenocarcinoma. Mod. Pathol. 21: 817-825.

Dwivedi PP, Lam N and Powell BC (2013). Boning up on glypicans - opportunities for new insights into bone biology. Cell Biochem. Funct. 31: 91-114.

Feng M and Ho M (2014). Glypican-3 antibodies: a new therapeutic target for liver cancer. FEBS Lett. 588: 377-382.

Gao W and Ho M (2011). The role of glypican-3 in regulating Wnt in hepatocellular carcinomas. Cancer Rep. 1: 14-19.

Greten TF, Duffy AG and Korangy F (2013). Hepatocellular carcinoma from an immunologic perspective. Clin. Cancer Res. 19: 6678-6685.

Kim H, Xu GL, Borczuk AC, Busch S, et al. (2003). The heparan sulfate proteoglycan GPC3 is a potential lung tumor suppressor. Am. J. Respir. Cell. Mol. Biol. 29: 694-701.

Kim YH and Kang JS (2013). Expression of glypican-3 in mouse embryo stem cells and its derived hepatic lineage cells treated with diethylnitrosamine in vitro. Asian Pac. J. Cancer Prev. 14: 6341-6345.

Komori H, Beppu T, Baba H, Nakatsura T, et al. (2010). Assessment of serum GPC3 as a tumor marker for hepatocellular carcinoma and malignant melanoma. Nihon Rinsho 68: 833-836.

Lin Q, Xiong LW, Pan XF, Gen JF, et al. (2012). Expression of GPC3 protein and its significance in lung squamous cell carcinoma. Med. Oncol. 29: 663-669.

Magistri P, Leonard SY, Tang CM, Chan JC, et al. (2013). The glypican 3 hepatocellular carcinoma marker regulates human hepatic stellate cells via Hedgehog signaling. J. Surg. Res. 187: 377-385.

Pez F, Lopez A, Kim M, Wands JR, et al. (2013). Wnt signaling and hepatocarcinogenesis: molecular targets for the development of innovative anticancer drugs. J. Hepatol. 59: 1107-1117.

Pilia G, Hughes-Benzie RM, MacKenzie A, Baybayan P, et al. (1996). Mutation s in GPC3, a glypican gene, cause the Simpson-Golabi-Behmel overgrowth syndrome. Nat. Genet. 12: 241-247.

Sakane H, Yamamoto H, Matsumoto S, Sato A, et al. (2012). Localization of glypican-4 in different membrane microdomains is involved in the regulation of Wnt signaling. J. Cell Sci. 125: 449-460.

Tanaka SS, Kojima Y, Yamaguchi YL, Nishinakamura R, et al. (2011). Impact of WNT signaling on tissue lineage differentiation in the early mouse embryo. Dev. Growth Differ. 53: 843-856.

Umezu T, Shibata K, Kajiyama H, Yamamoto E, et al. (2010). Glypican-3 expression predicts poor clinical outcome of patients with early-stage clear cell carcinoma of the ovary. J. Clin. Pathol. 63: 962-966.

Ushiku T, Uozaki H, Shinozaki A, Ota S, et al. (2009). Glypican 3-expressing gastric carcinoma: distinct subgroup unifying hepatoid, clear-cell, and alpha-fetoprotein-producing gastric carcinomas. Cancer Sci. 100: 626-632.

Xu C, Yan Z, Zhou L and Wang Y (2013). A comparison of glypican-3 with alpha-fetoprotein as a serum marker for hepatocellular carcinoma: a meta-analysis. J. Cancer Res. Clin. Oncol. 139: 1417-1424.

Xiang YY, Ladeda V and Filmus J (2001). Glypican-3 expression is silenced in human breast cancer. Oncogene 20: 74087412. 